\title{
Diffusion, dimensionality, and noise in transcriptional regulation
}

\author{
Gašper Tkačik ${ }^{1, *}$ and William Bialek ${ }^{1,2,3}$ \\ ${ }^{1}$ Joseph Henry Laboratories of Physics, Lewis-Sigler Institute for Integrative Genomics, \\ Princeton University, Princeton, New Jersey 08544, USA \\ ${ }^{2}$ Princeton Center for Theoretical Science, Princeton University, Princeton, New Jersey 08544, USA \\ ${ }^{3}$ Center for Studies in Physics and Biology, The Rockefeller University, 1230 York Avenue, New York, New York 10065, USA
}

(Received 19 December 2008; published 4 May 2009)

\begin{abstract}
The precision of biochemical signaling is limited by randomness in the diffusive arrival of molecules at their targets. For proteins binding to specific sites on DNA and regulating transcription, the ability of the proteins to diffuse in one dimension by sliding along the length of the DNA, in addition to their diffusion in bulk solution, would seem to generate a larger target for DNA binding, consequently reducing the noise in the occupancy of the regulatory site. Here we show that this effect is largely canceled by the enhanced temporal correlations in one-dimensional diffusion. With realistic parameters, sliding along DNA has surprisingly little effect on the physical limits to the precision of transcriptional regulation.
\end{abstract}

DOI: 10.1103/PhysRevE.79.051901

PACS number(s): 87.15.Vv, 87.16.Xa, 87.18.Tt

\section{INTRODUCTION}

Cells constantly regulate the expression levels of their genes. A central motif in this regulatory process is the binding of transcription factor proteins to specific sites along the DNA. The precision of transcriptional regulation is limited, ultimately, by randomness in the arrival of transcription factor (TF) molecules at these sites [1]. But proteins can find their binding sites on DNA by two very different mechanisms - either by diffusing in three dimensions (3D) through the surrounding solution, or by binding weakly and diffusing in one dimension (1D) along the contour of the DNA molecule. The idea of diffusion or sliding along DNA goes back (at least) to the realization that the lac repressor seems to bind more rapidly to its target site than would be allowed by three-dimensional diffusion alone [2,3]. The theoretical understanding of the association kinetics of TFs to their cognate sites in the DNA in the presence of $1 \mathrm{D}$ and 3D diffusion was developed by Berg and co-workers [4,5]. More recently, the discussion of $3 \mathrm{D}$ vs $1 \mathrm{D}$ diffusion has been revitalized by theoretical analysis of the optimal search strategies [6], by new biochemical measurements [7], and by direct physical observations of the sliding motion [8-10]. Here we consider the impact of dimensionality and diffusion on the physical limits to the precision of transcriptional regulation.

Any physical system which responds to the concentration of a signaling molecule will exhibit noise due to the random diffusion of these molecules in and out of its "sensitive volume" [1]; the larger the volume, the smaller the (fractional) noise. This leads to the intuition that $1 \mathrm{D}$ diffusion will have a huge impact on the noise because it effectively increases the size of the target to which the transcription factor is binding [11]. Our main result is that this intuition is wrong. The problem is that diffusion in one dimension has a very different statistical structure than in three dimensions, and so one

\footnotetext{
*Present address: Department of Physics and Astronomy, University of Pennsylvania, Philadelphia, PA 19104-6396.
}

cannot simply say that $1 \mathrm{D}$ sliding generates a larger 3D target. We show that realistic combinations of 3D and $1 \mathrm{D}$ diffusions in fact have a surprisingly small effect on the limiting noise level - the increased size of the target is largely canceled by stronger temporal correlations, which means that integrating over a fixed time gives fewer independent samples.

\section{CONCENTRATION FLUCTUATIONS}

Before starting on the problem of proteins binding to DNA, it is useful to recall some facts about concentration fluctuations for molecules free in solution [12]. If we write the concentration as $c(\mathbf{x}, t)$, then the power spectrum of fluctuations $S_{c}(\mathbf{k}, \omega)$ is defined by

$$
\left\langle\delta c(\mathbf{x}, t) \delta c\left(\mathbf{x}^{\prime}, t^{\prime}\right)\right\rangle=\int \frac{d^{d} \mathbf{k}}{(2 \pi)^{d}} \int \frac{d \omega}{2 \pi} S_{c}(\mathbf{k}, \omega) e^{+i \mathbf{k} \cdot\left(\mathbf{x}-\mathbf{x}^{\prime}\right)-i \omega\left(t-t^{\prime}\right)},
$$

where $d$ is the dimensionality of space. For molecules present at the mean concentration $\bar{c}$ and diffusing freely with diffusion constant $D$, the power spectrum is given by

$$
S_{c}(\mathbf{k}, \omega)=\frac{2 \bar{c} D k^{2}}{\left(D k^{2}\right)^{2}+\omega^{2}},
$$

where $k=|\mathbf{k}|$. This result is independent of the dimensionality $d$, although of course the units of concentration are different in different dimensions. If we integrate over all frequencies, which corresponds to making instantaneous measurements, we find a spatial power spectrum

$$
\int \frac{d \omega}{2 \pi} S_{c}(\mathbf{k}, \omega)=\bar{c}
$$

This is spatial white noise, and embodies the fact that a snapshot of molecules in solution will reveal a random, Poisson distribution: the variance of the molecule number in any finite volume is equal to the mean, so the power spectrum of concentration fluctuations is equal to the mean concentration, 
and again this is independent of dimensionality.

Consider a measurement of concentration that is averaged over some small region of space,

$$
C(t)=\int d^{d} \mathbf{x} W(\mathbf{x}) c(\mathbf{x}, t),
$$

where $W$ is a weighting or windowing function that defines the size of the region we are interested in. To interpret $C$ as an average of the local variable $c$, we must have $\int d^{d} \mathbf{x} W(\mathbf{x})$ $=1$. The temporal fluctuations in $C$ are themselves determined by a power spectrum $S_{C}(\omega)$,

$$
\begin{gathered}
\left\langle\delta C(t) \delta C\left(t^{\prime}\right)\right\rangle=\int \frac{d \omega}{2 \pi} S_{C}(\omega) e^{-i \omega\left(t-t^{\prime}\right)}, \\
S_{C}(\omega)=\int \frac{d^{d} \mathbf{k}}{(2 \pi)^{d}} S_{c}(\mathbf{k}, \omega)|\tilde{W}(\mathbf{k})|^{2},
\end{gathered}
$$

where

$$
\tilde{W}(\mathbf{k})=\int d^{d} \mathbf{x} W(\mathbf{x}) e^{i \mathbf{k} \cdot \mathbf{x}}
$$

The normalization of $W$ implies that $\tilde{W}(\mathbf{k}=0)=1$. If the size of the region is $\ell^{d}$, then $\tilde{W}(\mathbf{k})$ will fall to zero for $k$ much larger than $\Lambda \sim 1 / \ell$. Thus we can approximate

$$
\begin{aligned}
S_{C}(\omega) & =\int \frac{d^{d} \mathbf{k}}{(2 \pi)^{d}} \frac{2 \bar{c} D k^{2}}{\left(D k^{2}\right)^{2}+\omega^{2}}|\tilde{W}(\mathbf{k})|^{2} \\
& \sim \int_{0}^{\Lambda} d k k^{d-1} \frac{2 \bar{c} D k^{2}}{\left(D k^{2}\right)^{2}+\omega^{2}} .
\end{aligned}
$$

We are interested in the behavior of the power spectrum at low frequencies, corresponding to measurements with long averaging times. If we try simply to set $\omega=0$, then we find

$$
S_{C}(\omega=0) \sim \int_{0}^{\Lambda} d k k^{d-1} \frac{2 \bar{c}}{D k^{2}}
$$

For $d=3$,

$$
S_{C}^{3 d}(\omega=0) \sim \int_{0}^{\Lambda} d k k^{2} \frac{2 \bar{c}}{D k^{2}} \sim \frac{2 \bar{c} \Lambda}{D} \sim \frac{\bar{c}}{D \ell} .
$$

This corresponds to white noise in the time domain, so we expect that averaging over time will reduce the noise variance in proportion to the averaging time. More precisely, if we average for a time $\tau_{\text {int }}$, we will be sensitive to frequencies $|\omega|<1 / \tau_{\text {int }}$, so we will see a variance

$$
\begin{aligned}
\left\langle(\delta C)^{2}\right\rangle_{3 d} & \sim \int_{|\omega|<1 / \tau_{\mathrm{int}}} \frac{d \omega}{2 \pi} S_{C}^{3 d}(\omega) \\
& \sim \int_{|\omega|<1 / \tau_{\mathrm{int}}} \frac{d \omega}{2 \pi} S_{C}^{3 d}(\omega=0)
\end{aligned}
$$

$$
\sim \frac{\bar{c}}{D \ell \tau_{\text {int }}} \text {. }
$$

Although we have not done a full calculation including the effects of binding and unbinding to target sites, this is essentially the "noise floor" for a system which senses the concentration of signaling molecules by having them bind to a site of size $\ell[1,13,14]$.

In contrast, for $d=1$ we have

$$
S_{C}^{1 d}(\omega=0) \sim \int_{0}^{\Lambda} d k \frac{2 \bar{c}}{D k^{2}},
$$

which is divergent at small $k$. To get the right low-frequency behavior in one dimension we have to be a bit more careful. We have

$$
S_{C}^{1 d}(\omega) \sim \int_{0}^{\Lambda} d k \frac{2 \bar{c} D k^{2}}{\left(D k^{2}\right)^{2}+\omega^{2}} .
$$

This integral is actually finite as $\Lambda \rightarrow \infty$, so we can write

$$
\begin{aligned}
S_{C}^{1 d}(\omega) \sim & \int_{0}^{\infty} d k \frac{2 \bar{c} D k^{2}}{\left(D k^{2}\right)^{2}+\omega^{2}} \\
= & \frac{2 \bar{c}}{\sqrt{D \omega}} \int_{0}^{\infty} d\left(k \sqrt{\frac{D}{\omega}}\right) \\
& \times \frac{D k^{2} / \omega}{\left(D k^{2} / \omega\right)^{2}+1} \\
= & \frac{2 \bar{c}}{\sqrt{D \omega}} \int_{0}^{\infty} d q \frac{q^{2}}{q^{4}+1} \\
\sim & \frac{\bar{c}}{\sqrt{D \omega}} .
\end{aligned}
$$

Now the variance of measurements averaged over a time $\tau_{\text {int }}$ becomes

$$
\begin{aligned}
\left\langle(\delta C)^{2}\right\rangle_{1 d} & \sim \int_{|\omega|<1 / \tau_{\text {int }}} \frac{d \omega}{2 \pi} S_{C}^{1 d}(\omega) \\
& \sim \int_{|\omega|<1 / \tau_{\text {int }}} \frac{d \omega}{2 \pi} \frac{\bar{c}}{\sqrt{D \omega}} \\
& \sim \frac{\bar{c}}{\sqrt{D \tau_{\text {int }}}} .
\end{aligned}
$$

We see that in one dimension, the variance of concentration measurements declines only as the square root of the measurement time. This is in contrast to the three-dimensional case, where the standard deviation of the noise declines as the square root of time but the variance declines in direct proportion to the measurement time, as seen in Eq. (14).

In the following sections we expand on these general results by presenting a calculation that explicitly couples diffusion to the kinetics of binding and unbinding to or from a 
target site. However, because the target site dimensions are as small as a nanometer, there is concern about whether diffusionlike models are appropriate or not. We first point out that we are by no means the first to apply the diffusion equation down to the length scales characterizing individual molecules: in the chemical physics literature there are numerous examples where diffusion-limited association rates are computed within this framework, and even small deviations from the theory are taken seriously. For the general application to biomolecular context, see the review in Ref. [15]; a concrete case that couples $1 \mathrm{D}$ and $3 \mathrm{D}$ diffusions in an association problem for a lac transcription factor is derived in Ref. [4], and has been generalized to curved DNA geometry in Ref. [5].

With this remark in mind we note that our aim is to compute the equilibrium behavior of the occupancy of the binding site on long-time scales. We do not track the evolution of motion of a single molecule along the DNA, but actually imagine a number of molecules diffusing in the bulk and along the DNA. Our results will concern the noise in the $\omega$ $\rightarrow 0$ limit, or, more precisely, the noise averaged over times which are much longer than the characteristic time that the TF particle needs to diffuse over length scales of the receptor or sliding length. Concretely, the noise in gene regulation is generally averaged on the cell division or protein lifetime scale, both of which are in the range of at least minutes, while diffusion across a region $1 \mathrm{~nm}$ in size at $D$ $\sim 1 \mu \mathrm{m}^{2} / \mathrm{s}$ will take on the order of $1 \mu \mathrm{s}$, and will be comparatively short even if such region is bigger by 2 orders of magnitude. This means that-over the relevant timescale-we will be able to define an ensemble of particles that samples the spatial neighborhood of the binding site extremely well and will thus be justified in using the diffusion equations. We expect that our continuous approach is inappropriate for probing small distances at short times, but this is not relevant here.

\section{BINDING WITH 3D DIFFUSION}

If transcription factors bind specific sites on the DNA to control the rate of gene expression, then the noise in the regulated gene product will contain a contribution from the noise in the occupancy of the specific site. Here we want to compute this noise contribution, starting with the case where the transcription factors find their cognate site by 3D diffusion alone and progressing toward more complicated translocation strategies in the following sections.

In this section we briefly review the calculations of Ref. [13]. Consider a cell volume with an average concentration $\bar{c}$ of TFs present, and a binding site located at $\mathbf{x}_{0}=0$. The transcription factor molecules can diffuse with a bulk diffusion constant $D_{3}$, bind to the specific site at a rate $k_{+} c$, and dissociate back into the free solution at a rate $k_{-}$, according to the following set of equations:

$$
\frac{\partial c}{\partial t}=D_{3} \nabla^{2} c-\dot{n} \delta\left(\mathbf{x}-\mathbf{x}_{0}\right)
$$

$$
\dot{n}=k_{+} c\left(\mathbf{x}_{0}, t\right)(1-n)-k_{-} n \text {. }
$$

To determine the fluctuations in the binding site occupancy $n$, we linearize the equations about the mean occupancy $n(t)=\delta n(t)+\bar{n}$ with $\bar{n}=k_{+} \bar{c} /\left(k_{+} \bar{c}+k_{-}\right)$, and around the mean concentration, $c(\mathbf{x}, t)=\delta c(\mathbf{x}, t)+\bar{c}$, and write the perturbations as Fourier modes,

$$
\begin{gathered}
\delta n(t)=\int \frac{d \omega}{(2 \pi)} e^{-i \omega t} \delta \widetilde{n}(\omega), \\
\delta c(\mathbf{x}, t)=\int \frac{d \omega}{(2 \pi)} \int \frac{d^{3} k}{(2 \pi)^{3}} e^{-i(\omega t+\mathbf{k} \cdot \mathbf{x})} \delta \widetilde{c}_{\mathbf{k}}(\omega) .
\end{gathered}
$$

We are looking for the power spectrum of fluctuations in occupancy, which we can define by

$$
\left\langle\delta \widetilde{n}(\omega) \delta \widetilde{n}^{*}\left(\omega^{\prime}\right)\right\rangle=2 \pi \delta\left(\omega-\omega^{\prime}\right) S_{n}(\omega) .
$$

If there exists a low-frequency limit, $S_{n}(\omega \rightarrow 0)$, and we average the noise for a time $\tau_{\text {int }}$ that is longer than all other relevant timescales in the problem, we can [by analogy with the arguments leading to Eq. (14)] use it to calculate the observable variance by computing $\sigma_{n}^{2}=S_{n}(\omega \rightarrow 0) / \tau_{\text {int }}$.

Having introduced the necessary notation, we can compute the fluctuations in concentration at the binding site, $\mathbf{x}_{0}$ $=0$, from Eq. (24),

$$
\delta \widetilde{c}\left(\mathbf{x}_{0}, \omega\right)=i \omega \delta \tilde{n} \int \frac{d^{3} k}{(2 \pi)^{3}} \frac{1}{-i \omega+D_{3} k^{2}} .
$$

This integral is ultraviolet divergent, and must be cut off at $\Lambda=\frac{\pi}{a}$, where $a$ is the binding site size; keeping leading terms in $i \omega$ one obtains,

$$
\delta \widetilde{c}\left(\mathbf{x}_{0}, \omega \rightarrow 0\right)=\frac{i \omega \delta \tilde{n}}{2 \pi D_{3} a}
$$

In linearizing Eq. (25), we should take account of the possibility that the rates $k_{ \pm}$themselves are fluctuating; but as explained in Ref. [13], these fluctuations are constrained by detailed balance, $k_{+} / k_{-} \propto \exp \left(F / k_{B} T\right)$, where $F$ is the freeenergy difference between the bound and empty states of the site (the binding energy). The result is that

$$
-i \omega \delta \tilde{n}=-\frac{\delta \tilde{n}}{\tau_{c}}+k_{+}(1-\bar{n}) \delta \widetilde{c}\left(\mathbf{x}_{0}, \omega\right)+k_{-} \bar{n} \beta \delta \tilde{F},
$$

where $\tau_{c}^{-1}=k_{+} \bar{c}+k_{-}$is the time scale of occupancy fluctuations, $\beta=1 / k_{B} T$, and $\delta \tilde{F}$ is the fluctuation in the binding energy; this is the "force" that is thermodynamically conjugate to the "displacement" $\delta n$; for details see Ref. [13].

After substituting the local concentration fluctuations, Eq. (30), into Eq. (31), one can finally use the fluctuationdissipation theorem to compute the power spectrum of the noise in occupancy, 


$$
S_{n}(\omega)=\frac{2 k_{B} T}{\omega} \operatorname{Im} \frac{\delta \tilde{n}}{\delta \widetilde{F}} .
$$

The result of Ref. [13] was that, in the case of 3D diffusion to the binding site, the low-frequency limit of the occupancy noise power spectrum is

$$
S_{n}(\omega \rightarrow 0)=\frac{2 \bar{n}(1-\bar{n})^{2}}{k_{-}}+\frac{\bar{n}^{2}(1-\bar{n})^{2}}{\pi D_{3} a \bar{c}} .
$$

The first term corresponds to the binomial switching fluctuations as the occupancy of the specific site changes between full and empty; this term depends on the microscopic details of the TF-DNA interaction (here embodied by the off-rate constant $\left.k_{-}\right)$. The second term is caused by the fluctuating diffusive flux to the binding site, and provides a lower bound on the noise, independent of details. Expressed in terms of the equivalent concentration fluctuations, $S_{c}(\omega)=S_{n}(\omega)\left|\frac{d \bar{n}}{d c}\right|^{-2}$, the noise spectrum for the second term is

$$
S_{c}(\omega \rightarrow 0)=\bar{c} / \pi D_{3} a,
$$

with the associated fractional variance

$$
\left(\frac{\sigma_{c}}{\bar{c}}\right)^{2}=\frac{1}{\pi} \times \frac{1}{D_{3} \bar{c} \tau_{\text {int }}} \times \frac{1}{a},
$$

where, to facilitate comparison with results that follow, we explicitly pull out the leading numerical factor and $1 /$ (effective binding site size). This is the noise lower bound, consistent with the result of Eq. (14) for $\ell=a$, that depends solely on the rate of 3D diffusion $D_{3}$, the binding site size $a$, and the amount of time averaging that the system performs, $\tau_{\text {int }}$.

\section{BINDING WITH 1D DIFFUSION}

Consider the case where the diffusion in bulk does not occur, and the TF is only free to slide along the length of the DNA. Let $\xi(x, t)$ denote the $1 \mathrm{D}$ concentration along the DNA contour, which we imagine to be stretched in the $\hat{\mathbf{x}}$ direction, and let $D_{1}$ be the corresponding diffusion constant. Equations (24) and (25) remain unchanged but for the new notation, i.e., $c(\mathbf{x}, t) \rightarrow \xi(x, t), D_{3} \rightarrow D_{1}, \quad \nabla^{2} \rightarrow \partial_{x}^{2}$; we will use $q$ to denote the Fourier variable conjugate to $x$. We again need to calculate [as in Eq. (29)] the change in concentration at the binding site in response to fluctuations in the occupancy of the site itself, and we find

$$
\delta \tilde{\xi}\left(x_{0}, \omega\right)=i \omega \delta \tilde{n} \int_{-\infty}^{\infty} \frac{d q}{(2 \pi)} \frac{1}{-i \omega+D_{1} q^{2}} .
$$

This integral diverges as $q \rightarrow 0$ if we set $\omega=0$, but in contrast to the discussion above there is no problem as $q \rightarrow \infty$, echoing the results for the simpler problem in Sec. II. The full result is that

$$
\delta \tilde{\xi}\left(x_{0}, \omega\right)=\frac{i \omega \delta \tilde{n}}{2 D_{1} \sqrt{-i \omega / D_{1}}} .
$$

Note that unlike Eq. (30), this result does not contain the binding site dimensions. Using Eq. (37) we compute the dif- fusive contribution to the noise power spectrum [cf. Eq. (34), but no limit $\omega \rightarrow 0$ ],

$$
S_{\xi}(\omega)=\frac{\bar{\xi}}{\sqrt{2 \omega D_{1}}} .
$$

The noise variance is obtained by integrating the noise power spectrum, as in the arguments leading to Eq. (23),

$$
\left(\frac{\sigma_{\xi}}{\bar{\xi}}\right)^{2}=\frac{2}{\pi} \frac{1}{\sqrt{2 D_{1} \tau_{\text {int }}} \bar{\xi}} .
$$

We see that the noise variance declines as $1 / \sqrt{\tau_{\text {int }}}$, consistent with the simpler calculation of concentration fluctuations in Eq. (23). This again is in contrast to our intuition that variances should decline as $1 / \tau_{\text {int }}$, which is the result for binding coupled to 3D diffusion. The difference between 1D and 3D can be understood by realizing that in 1D diffusion, a particle leaving the binding site at the origin has a large probability (in fact, probability 1) of returning back to the origin. A receptor trying to estimate the local concentration of TFs will therefore be unable to collect samples that are truly independent, and the variance in the measurements will consequently decrease at a rate that is slower than expected from averaging over independent measurements.

Suppose that the DNA with the sliding TFs is embedded into the cytoplasm, where the bulk concentration of TFs is $\bar{c}$; the TFs can jump onto the DNA at a rate (per unit length) $\kappa_{+} \bar{c}$, where we expect $\kappa_{+} \sim D_{3}$. The TF will stay on the strand for a residence time, $\tau_{\mathrm{r}}=\kappa_{-}^{-1}$, where $\kappa_{-}$is the rate for dissociating from the DNA. There will be an equilibrium between the $1 \mathrm{D}$ and $3 \mathrm{D}$ concentrations, $\bar{\xi}=\kappa_{+} \tau_{\mathrm{r}} \bar{c}$, so that $\bar{c} \approx \bar{\xi} / \tau_{\mathrm{r}} D_{3}$. With these identifications, the noise variance can be rewritten in a form similar to the result in Eq. (35),

$$
\left(\frac{\sigma_{c}}{\bar{c}}\right)^{2}=\frac{\sqrt{2}}{\pi} \times \frac{1}{D_{3} \bar{c} \tau_{\mathrm{int}}} \times \frac{1}{\sqrt{D_{1} \tau_{\mathrm{r}}^{2} / \tau_{\mathrm{int}}}} .
$$

Here, the effective binding site size is $a_{\text {eff }}=\sqrt{D_{1} \tau_{\mathrm{r}}} \sqrt{\tau_{\mathrm{r}} / \tau_{\text {int }}}$. Naively, one could think that the effective site size would be equal to the average length that the TF explores during $1 \mathrm{D}$ diffusion on the DNA, $b=\sqrt{D_{1} \tau_{\mathrm{r}}}$, and therefore $a_{\mathrm{eff}} \sim b$, but that would be wrong: the effective site size depends on $\tau_{\text {int }}$ to compensate for the highly correlated fluctuations in 1D diffusion. Because $\tau_{\text {int }} \gg \tau_{\mathrm{r}}$ [16], the effective site size will be much smaller than $b$. The reduction in the noise expected in the naive picture-because the size of the binding site, $a$, is replaced by presumably much larger length explored by 1D diffusion, $b$-must in reality be traded off against the longer required integration time. As a result, it is not immediately clear whether 1D diffusion decreases the noise lower bound compared to 3D case.

\section{COMBINED 1D AND 3D DIFFUSION}

In the discussion so far we have been missing a parameter that would interpolate between two qualitatively different noise regimes: the $3 \mathrm{D}$ result of Eq. (35) and the $1 \mathrm{D}$ result of Eq. (40). If the rate for dissociation from the sliding state 
into the bulk is increased, the $1 \mathrm{D}$ case must approach the $3 \mathrm{D}$ result; the residence time will grow ever shorter and will, at some scale, break the strong correlations reflective of the $1 \mathrm{D}$ sliding mode with its high probability for returning to the origin.

\section{A. Perfect mixing in bulk solution}

We first solve a simplified case where the TF diffuses on the DNA, but if it dissociates into the bulk, it mixes very quickly and there is no correlation between the point of dissociation and subsequent reassociation to the DNA strand. We expect that the divergence observed in the pure 1D case, Eq. (36), will now get regularized. The dynamical equations for this case are

$$
\begin{gathered}
\frac{d n}{d t}=k_{+} \xi\left(x_{0}, t\right)(1-n)-k_{-} n, \\
\frac{\partial \xi}{\partial t}=D_{1} \frac{\partial^{2} \xi}{\partial x^{2}}-\dot{n} \delta\left(x-x_{0}\right)-\kappa_{-} \xi+\kappa_{+} C, \\
\frac{d C}{d t}=\kappa_{-} \int d x \xi(x, t)-\kappa_{+} L C .
\end{gathered}
$$

Here, $C(t)$ is the bulk concentration that is perfectly mixed and therefore does not have any spatial dependence; TFs in the bulk are enclosed into a box with a side of length $L$ and the DNA inside is an infinitely thin line of length $L$; and $\kappa_{-}$ and $\kappa_{+}$are rates for transitioning between 1D concentration $\xi$ and the bulk $C$. When linearized and written out in terms of their Fourier components, the equations couple to the zero mode, $\delta \tilde{\xi}(q=0, \omega)$, differently than to nonzero modes,

$$
\begin{gathered}
\left(-i \omega+1 / \tau_{c}\right) \delta \tilde{n}=k_{+}(1-\bar{n}) \delta \tilde{\xi}\left(x_{0}\right)+k_{-} \bar{n} \beta \delta F, \\
\left(-i \omega+D_{1} q^{2}\right) \delta \tilde{\xi}_{q}=i \omega \delta \tilde{n}-\kappa_{-} \delta \tilde{\xi}_{q}+\kappa_{+} L \delta \tilde{C}, \\
\left(-i \omega+\kappa_{+} L\right) \delta \tilde{C}=\kappa_{-} \delta \tilde{\xi}_{q=0} .
\end{gathered}
$$

We use Eq. (45) at $q=0$ together with Eq. (46) to express $\delta \tilde{C}$ and substitute it back into Eq. (45) for nonzero $q$; it is then straightforward to write $\delta \tilde{\xi}\left(x_{0}\right)$ as

$$
\begin{aligned}
\delta \xi\left(x_{0}\right) & =\int \frac{d q}{(2 \pi)} \frac{i \omega \Sigma(\omega) \delta \tilde{n}}{-i \omega+D_{1} q^{2}+\kappa_{-}} \\
& =\frac{i \omega \Sigma(\omega)}{2 \sqrt{D_{1}}} \frac{\delta \tilde{n}}{\sqrt{\kappa_{-}-i \omega}},
\end{aligned}
$$

where

$$
\Sigma(\omega)=1+\frac{\kappa_{-} \kappa_{+} L}{\left(-i \omega+\kappa_{-}\right)\left(-i \omega+\kappa_{+} L\right)-\kappa_{-} \kappa_{+} L} .
$$

Note that as $\omega \rightarrow 0, \Sigma(\omega)$ will diverge; before computing the power spectrum of fluctuations $S_{n}$ using Eq. (32) we therefore need to expand Eq. (47) to the leading power of $i \omega$. On the other hand, the $q$ integral in Eq. (47) now converges due to the $\kappa_{-}$term in the denominator.

After some algebra, the effective power spectrum in the concentration fluctuations is

$$
S_{\xi}(\omega \rightarrow 0)=\frac{\bar{\xi}}{2 \sqrt{D_{1} \kappa_{-}}}\left(1+\frac{1-\gamma}{(1+\gamma)^{2}}\right),
$$

where $\gamma=\kappa_{+} L / \kappa_{-}$is the ratio of on and off rates for going into 1D solution. The $\gamma$-dependent expression in parenthesis has a minimum of $7 / 8$ at $\gamma_{0}=3$, and therefore a noise floor exists that cannot be eliminated by some convenient combination of the rate parameters.

Rewritten in terms of the "equivalent concentration" $\bar{c}$ $=\bar{C} / L^{3}=\kappa_{-} \bar{\xi} / \kappa_{+} L^{3} \approx \kappa_{-} \bar{\xi} / D_{3}$, the concentration noise floor is [cf. Eq. (40)],

$$
\left(\frac{\sigma_{c}}{\bar{c}}\right)^{2}=\frac{7}{16} \times \frac{1}{D_{3} \bar{c} \tau_{\mathrm{int}}} \times \frac{1}{\sqrt{D_{1} \tau_{\mathrm{r}}}} .
$$

This is the result we might naively have expected, namely, that sliding along the DNA creates a larger target, of size $a_{\mathrm{eff}} \approx b$. But we get this result only because we have assumed that the TF can repeatedly dissociate into a well-mixed bulk solution, instantly losing the memory of the point along the DNA from which it dissociated.

\section{B. Diffusion in bulk solution}

We will now solve the coupled 3D-1D diffusion problem without assuming that in the bulk solution the transcription factors mix perfectly. The relevant quantities are schematized in Fig. 1.

We describe the system by the following set of equations:

$$
\begin{aligned}
& \frac{d n}{d t}=k_{+} \xi\left(x_{0}, t\right)(1-n)-k_{-} n, \\
& \frac{\partial \xi(x, t)}{\partial t}=D_{1} \frac{\partial^{2} \xi(x, t)}{\partial x^{2}}-\frac{d n}{d t} \delta\left(x-x_{0}\right) \\
& +\kappa_{+} c(x, \mathbf{R}=\mathbf{0}, t)-\kappa_{-} \xi(x, t), \\
& \frac{\partial c(x, \mathbf{R}, t)}{\partial t}=D_{3} \nabla^{2} c(x, \mathbf{R}, t)-\left[\kappa_{+} c(x, \mathbf{R}=\mathbf{0}, t)\right. \\
& \left.-\kappa_{-} \xi(x, t)\right] \delta(y) \delta(z) .
\end{aligned}
$$

We have again assumed that DNA is stretched along the $x$ axis and that it is an infinitely thin molecule. $\xi$ is a function of only one variable, $x$, while $c$ is a function of coordinate $x$ and radial coordinates $\mathbf{R}$ that are perpendicular to the $\hat{\mathbf{x}}$ direction.

We linearize and Fourier transform the equations as follows:

$$
\begin{gathered}
-i \omega \delta \tilde{n}=-\frac{\delta \tilde{n}}{\tau_{c}}+k_{+}(1-\bar{n}) \delta \tilde{\xi}\left(x_{0}\right)+k_{-} \bar{n} \beta \delta \tilde{F}, \\
-i \omega \delta \tilde{\xi}_{q}=-D_{1} q^{2} \delta \tilde{\xi}_{q}+i \omega \delta \tilde{n}-\kappa_{-} \delta \tilde{\xi}_{q}+\kappa_{+} \delta \hat{c}_{q},
\end{gathered}
$$




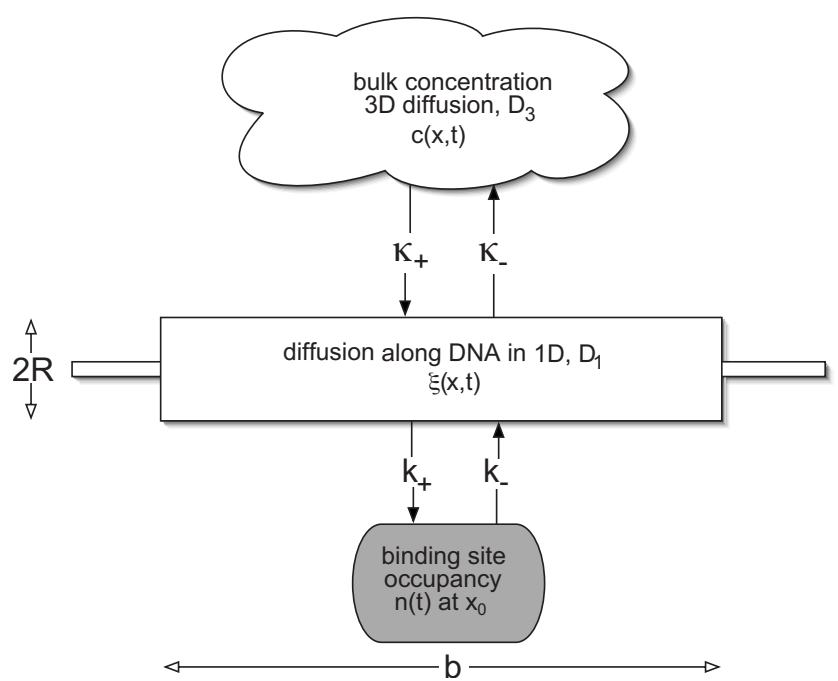

FIG. 1. The transcription factors can either be free in solution at concentration $c$, or they can enter a region on the DNA where they diffuse by sliding. The 1D concentration is denoted by $\xi(x, t)$. The specific binding site on the DNA is at location $x_{0}=0 ; k_{+} \xi\left(x_{0}\right)$ and $k_{-}$ are the on and off rates for transitions between the 1D sliding state and the bound state on the specific site; $\kappa_{+} c$ is the rate per unit length for transition onto the DNA from free solution, and $\kappa_{-}=\tau_{\mathrm{r}}^{-1}$ is the rate for dissociating from $1 \mathrm{D}$ solution into the $3 \mathrm{D}$ solution. The effective radius of the DNA molecule is $R$ and the "sliding length," or the average distance along the contour covered in a single 1D random walk before dissociation, is $b$.

$$
-i \omega \delta \widetilde{c}_{q, \mathbf{k}_{\perp}}=-D_{3}\left(q^{2}+k_{\perp}^{2}\right) \delta \widetilde{c}_{q, \mathbf{k}_{\perp}}+\kappa_{-} \delta \tilde{\xi}_{q}-\kappa_{+} \delta \hat{c}_{q},
$$

where again $q$ is the spatial Fourier variable conjugate to $x$ and $\mathbf{k}_{\perp}$ is conjugate to $\mathbf{R}$. Note that $\delta \widetilde{\xi}_{q}$ is function only of $q$, while $\delta \widetilde{c}_{q, \mathbf{k}_{\perp}}$ is a 3D Fourier transform of the bulk concentration fluctuations that depends on both $q$ and $\mathbf{k}_{\perp}$. Finally, $\delta \hat{c}(q, \mathbf{R}=\mathbf{0}, \omega)$ are Fourier modes of concentration fluctuations along the $\hat{\mathbf{x}}$ direction, evaluated at the location of the DNA strand, $\mathbf{R}=\mathbf{0}$.

We first express $\delta \widetilde{c}_{q, \mathbf{k}}$ from Eq. (57),

$$
\delta \widetilde{c}_{q, \mathbf{k}_{\perp}}=\frac{\kappa_{-} \delta \tilde{\xi}_{q}-\kappa_{+} \delta \hat{c}_{q}}{-i \omega+D_{3} q^{2}+D_{3} k_{\perp}^{2}} .
$$

The quantity $\delta \hat{c}_{q}$ from Eq. (56) is an integral of $\delta \widetilde{c}_{q, \mathbf{k}_{\perp}}$ over momenta $\mathbf{k}_{\perp}$,

$$
\begin{aligned}
\delta \hat{c}_{q} & =\int \frac{d^{2} k_{\perp}}{(2 \pi)^{2}} \delta \widetilde{c}_{q, \mathbf{k}_{\perp}} \\
& =\int \frac{d^{2} k_{\perp}}{(2 \pi)^{2}} \frac{\kappa_{-} \delta \tilde{\xi}_{q}-\kappa_{+} \delta \hat{c}_{q}}{D_{3} q^{2}+D_{3} k_{\perp}^{2}} \\
& =\frac{\kappa_{-} \delta \tilde{\xi}_{q}-\kappa_{+} \delta \hat{c}_{q}}{4 \pi D_{3}} \log \left\{1+\frac{\Lambda^{2}}{k_{0}^{2}}\right\} .
\end{aligned}
$$

Here, $k_{0}^{2}=q^{2}-i \omega / D_{3}$, and $\Lambda=\frac{\pi}{R}$ is the cutoff at the (inverse) radial size of the DNA molecule, as in Eq. (29). We can substitute $\delta \hat{c}_{q}$ from Eq. (61) into Eq. (56) to obtain the expression for $\delta \widetilde{\xi}\left(x_{0}\right)$,

$$
\begin{gathered}
\delta \tilde{\xi}\left(x_{0}\right)=i \omega \delta \tilde{n} \int \frac{d q}{(2 \pi)} \frac{1}{-i \omega+D_{1} q^{2}+\kappa_{-} F^{-1}(q)}, \\
F(q)=1+\frac{\kappa_{+}}{4 \pi D_{3}} \log \left\{1+\frac{\Lambda^{2}}{-i \omega / D_{3}+q^{2}}\right\} .
\end{gathered}
$$

Compared to the pure 1D result, Eq. (36), Eq. (62) now contains a new term $\kappa_{-} F^{-1}(q)$ in the denominator; for $\kappa_{-}$ $=0$ this term vanishes and the result reverts to the $1 \mathrm{D}$ case as expected. $F(q)$ depends on the momentum $q$, whereas in the perfect mixing case, Eq. (47), it was simply equal to 1 . The integrand of Eq. (62) is still divergent for $\omega=0$ as $q \rightarrow 0$, but the integral nevertheless converges. Assuming a nonzero $\kappa_{-}$, the limit $\omega \rightarrow 0$ therefore exists, and we can integrate

$$
\delta \tilde{\xi}\left(x_{0}, \omega \rightarrow 0\right)=\frac{i \omega \delta \tilde{n}}{\pi \Lambda D_{1}} I(\alpha, \beta),
$$

where

$$
\begin{gathered}
I(\alpha, \beta)=\int_{0}^{\infty} \frac{d t}{t^{2}+\beta\left[1+\alpha \log \left(1+t^{-2}\right)\right]^{-1}}, \\
\alpha=\frac{\kappa_{+}}{4 \pi D_{3}}, \\
\beta=\frac{\kappa_{-}}{\Lambda^{2} D_{1}}=\left(\frac{R}{\pi b}\right)^{2} .
\end{gathered}
$$

The noise power spectrum for occupancy follows in close analogy to Eq. (33),

$$
S_{n}(\omega \rightarrow 0)=\frac{2 \bar{n}(1-\bar{n})^{2}}{k_{-}}+\frac{2 \bar{n}^{2}(1-\bar{n})^{2}}{\pi \Lambda D_{1} \bar{\xi}} I(\alpha, \beta) .
$$

The effective spectrum of local concentration fluctuations corresponding to the second term is [cf. Eqs. (34), (38), and (50)]:

$$
S_{c}(\omega \rightarrow 0)=\frac{2 \bar{\xi} R}{\pi^{2} D_{1}} I(\alpha, \beta) .
$$

The associated noise variance is

$$
\left(\frac{\sigma_{\xi}}{\bar{\xi}}\right)^{2}=\frac{2 I(\alpha, \beta)}{\pi^{2} D_{1} \tau_{\mathrm{int}} \bar{\xi} / R} .
$$

With the above definition of $\alpha$, the equilibrium between 1D and $3 \mathrm{D}$ concentrations turns out to be $\bar{c}=\bar{\xi} / 4 \pi D_{3} \alpha \tau_{\mathrm{r}}$. The noise variance in concentration can be rewritten as

$$
\left(\frac{\sigma_{c}}{\bar{c}}\right)^{2}=\frac{\beta I(\alpha, \beta)}{2 \pi \alpha} \times \frac{1}{D_{3} \bar{c} \tau_{\text {int }}} \times \frac{1}{R} .
$$

The result looks similar to the pure 3D diffusion case in Eq. (35): the noise due to concentration fluctuations has its 
length scale $a$ (receptor size in pure 3D case) replaced with $R$ (effective DNA cross section in the combined 1D and 3D case), and the noise contribution term gets multiplied by the prefactor that we examine next.

We expect the parameter $\alpha=\kappa_{+} / 4 \pi D_{3}$ to be close to 1 for diffusion-limited approach to DNA. Imagine that the area that TF attempts to hit in order to bind nonspecifically to the DNA is a cylindrical segment of DNA with radius $R$ and length $b$. If we were treating the cylindrical DNA segment as a sphere of radius $b$, then Smoluchowski on-rate limit $\sim 4 \pi D_{3} b$ would apply [17]. In the first approximation, the on rate per unit length, $\kappa_{+}$, would then be $4 \pi D_{3}$ and $\alpha=1$. Exact derivations for Smoluchowski limit in simple geometries were reviewed in Ref. [15]; for elongated objects (prolate ellipsoid with long semiaxis $b$ and short semiaxis $R$ ), the diffusion-limited rate is given by $4 \pi D_{3} b / \ln (2 b / R)$, and therefore $\alpha \sim \ln ^{-1}(2 b / R) . \alpha$ is hence less then one, and has a weak logarithmic dependence on the ratio of the scale of the persistence length and the effective radius of the DNA.

The parameter $\beta$ is approximately the square of the ratio between the cross section of the 1D cylinder (the "target" that 3D diffusion has to hit) and the average "sliding length" $b$ along the DNA. $R$ must be of order of several nanometers; while $b$ is, at DNA stacking length of $a=0.3 \mathrm{~nm}$ per base pair and $100 \mathrm{bp}$ average diffusion length [6,22], around $b$ $=10-100 \mathrm{~nm}$. It is therefore not unreasonable to assume that the factor $\beta$ could be as low as $\beta \sim 10^{-3}-10^{-2}$. The corresponding decrease in noise relative to pure $3 \mathrm{D}$ diffusion is shown in Fig. 2. Importantly, note that for values of $\beta$ spanning 4 orders of magnitude, the noise reduction spans a range of only $\sim 5$.

Note that as $\beta \rightarrow \infty$ one should recover the pure 3D diffusion result. Looking back at expression for $I(\alpha, \beta)$ in Eq. (65), as $\beta$ increases, the $t^{2}$ term in the denominator of the integrand is becoming irrelevant. If we neglect it completely, the integral is solvable analytically,

$$
I(\alpha, \beta \rightarrow \infty)=\beta^{-1}\left(\frac{\Lambda_{x}}{\Lambda}+\pi \alpha\right),
$$

where $\Lambda_{x}=\frac{\pi}{a}$ is the cutoff at the binding site size along the DNA, and $\Lambda_{x} / \Lambda=R / a$. Inserting the integral in the large $\beta$ limit into the noise result, Eq. (71), we see that $\beta$ cancels and we get

$$
\begin{aligned}
\left(\frac{\sigma_{c}}{\bar{c}}\right)^{2} & =\frac{1}{2 \pi}\left(\pi+\frac{R}{\alpha a}\right) \times \frac{1}{D_{3} \bar{c} \tau_{\text {int }}} \times \frac{1}{R} \\
& >\frac{1}{2 \pi \alpha} \times \frac{1}{D_{3} \bar{c} \tau_{\text {int }}} \times \frac{1}{a},
\end{aligned}
$$

which is essentially the 3D result, Eq. (35). This limiting behavior is also clearly evident in Fig. 2, where the thick lines are plotted with momentum cutoff $\Lambda_{x} / \Lambda=a / R=1$ [which corresponds to the upper limit of integration in Eq. (65) at $t=1]$, and the thin lines have $\Lambda_{x} \rightarrow \infty$. In the biologically relevant range for parameter $\beta$, the two results do not differ appreciably, making our conclusions robust with respect to the precise value chosen for the cutoffs.

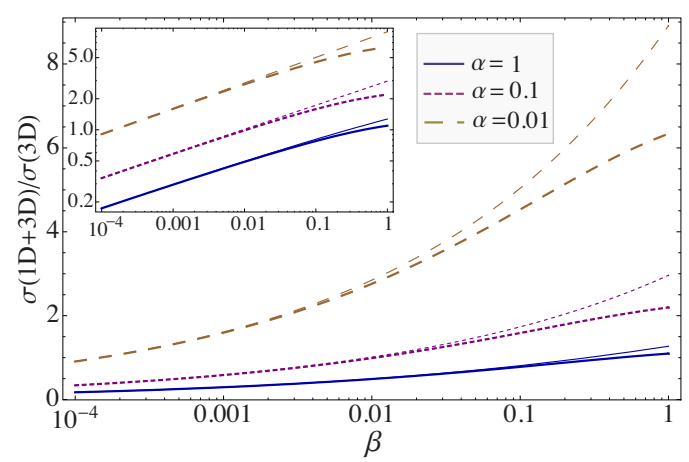

FIG. 2. (Color online) Effective concentration noise level, compared to the pure 3D diffusion model, as a function of parameters $\alpha$ and $\beta$. Parameter $\alpha=\kappa_{+} / 4 \pi D_{3}$ is the per unit length on rate for binding nonspecifically to the DNA, normalized by the Smoluchowski diffusion limit. Parameter $\beta=(R / \pi b)^{2}$ is approximately the square of the ratio between the radius of the DNA cylinder and the typical sliding length during a 1D diffusion excursion along the DNA contour length; in this plot it covers the relevant range if typical 1D diffusion length is as expected from search time optimality arguments (order hundred base pairs). Three values for $\alpha$ are shown, spanning 2 orders of magnitude ( $\alpha=1$ solid, $\alpha=0.1$ dotted, $\alpha=0.01$ dashed). For each value of $\alpha$, two curves are plotted: the thin curve with $\Lambda_{x} / \Lambda=R / a \rightarrow \infty$, and the thick curve with $\Lambda_{x} / \Lambda$ $=1$ (thin curves at given $\alpha$ have larger effective noise). The latter case in $\beta \rightarrow \infty$ (short sliding length) limit converges to the pure 3D diffusion result, as explained in Eq. (74). Importantly, note that while in both cases $\beta$ extends over 4 orders of magnitude, the relative decrease in noise is only of order 1 .

\section{DISCUSSION}

One-dimensional diffusion of transcription factors along the DNA has long been recognized as a possible explanation for the observed speed with which transcription factors find their targets. In contrast, the impact of this additional mode of TF translocation on the noise in binding site occupancy has remained unexplored. The question is important for two reasons: first, the diffusive contribution to the noise in gene expression must fundamentally limit the precision of transcriptional regulation; and second, there is an appealing argument that 1D diffusion could drastically increase the target area on the DNA that TFs have to find and correspondingly lower the limiting diffusive noise. Here we show that this intuitive argument is wrong-while there might be some reduction in the noise if the bulk diffusion is supplemented by 1D sliding, this reduction is not expected to be significant.

Much has been said about possible TF translocation strategies on the DNA, such as three-dimensional volume exchange, local dissociation-reassociation reactions (hopping), sliding along the DNA and intersegmental transfers mediated by DNA looping $[3,18]$, and about the ways in which these mechanisms influence the target search times [6,19-21]. While our model does not examine all of the proposed mechanisms, we are ultimately only interested in what happens locally around the specific site, as opposed to computing global properties such as target search time statistics. As a result, while rare but long-range excursions might be important in search arguments, they should not significantly 
affect the noise as long as they are not the dominant form of all bulk transfers; if they are, one would have the case discussed in Sec V A of perfect mixing upon dissociation. In our noise calculations we also make rather crude approximations to the DNA geometry, assuming it is a straight cylinder and neglecting the fluctuations in curvature. However, if the search optimality arguments in Refs. [6,22] are valid, then the 1D sliding lengths are on the order of or less than a persistence length, and a straight rod approximation should be sufficient for modeling a sliding diffusion excursion around the binding site. Moreover, fluctuations that in the extreme can lead to ring closure and facilitated TF intersegment transport, happen on a time scale for which an upperbound estimate of $\sim 4 \times 10^{-3} \mathrm{~s}$ is given in Refs. [3,23]; this is longer than an estimated time before dissociation in the optimal search scenario [22]. Without actual measurements of the relevant time and length scales it is clearly impossible to rule out the effects of local fluctuations of DNA on the 1D sliding process, but current estimates are at least consistent with the idea that, locally, a straight rod approximation is sufficient.

Despite much recent progress, experimental measurements of 3D and 1D diffusion constants, the residence time $\tau_{r}$ and even the absolute in vivo concentrations still remain a challenge. In Escherichia coli, for instance, the GFP diffuses with $D_{3} \sim 7 \mu \mathrm{m}^{2} / \mathrm{s}$, but this apparent diffusion constant can be substantially reduced by a small change in the protein [24]; the in vivo diffusion constant of lac was measured to be $\sim 3 \mu \mathrm{m}^{2} / \mathrm{s}$ [10]. In the Drosophila embryo, the in vivo diffusion constant of the Bicoid-GFP fusion was measured using fluorescence recovery after photobleaching to be as low as $D_{3} \sim 0.3 \mu \mathrm{m}^{2} / \mathrm{s}$ [25]. 1D diffusion constants seem even more elusive. On the one hand, based on a simple dimensional analysis one could argue that $D_{1} \sim D_{3}$, corrected for the possible interactions between the TF and the DNA that generate "frictional effects": this could reduce $D_{1}$ by about an order of magnitude from $D_{3}$ based on theoretical arguments [6]. Direct measurements, on the other hand, often exhibit smaller than expected 1D diffusion constants. For lac, for instance, the estimate is $D_{1} \sim 0.05 \mu \mathrm{m}^{2} / \mathrm{s}$ [10] (or $D_{1} \sim 0.02 \mu \mathrm{m}^{2} / \mathrm{s}$, with a large spread, in Ref. [9]); for RNA polymerase in Escherichia coli $D_{1} \sim 0.15 \mu \mathrm{m}^{2} / \mathrm{s}$ [26]. In contrast, the DNA repair protein hOgg1 seems to diffuse along the DNA with $D_{1} \sim 0.5 \mu \mathrm{m}^{2} / \mathrm{s}$, with $D_{1}$ depending strongly on the $p \mathrm{H}$ [27]. The 1D diffusion constant and the DNA residence time $\tau_{\mathrm{r}}$ (or, equivalently, the sliding off-rate $\left.\kappa_{-}\right)$combine into $\beta$, which directly affects both the absolute value of diffusion noise, as well as the relative noise in our $(1 \mathrm{D}+3 \mathrm{D})$ vs pure $3 \mathrm{D}$ comparison, plotted in Fig. 2 . In the light of these remarks, one of our main findings is therefore not only that the relative decrease in noise with added 1D diffusion is small for a particular value of $\beta$, but for a range in $\beta$ spanning 4 orders of magnitude.
To summarize, while our calculations are based on a number of simplifying assumptions, our conclusions nevertheless reflect the basic differences in the diffusive processes in one and three dimensions, in particular the high probability of returning to the origin in $1 \mathrm{D}$ diffusion. The conclusions are therefore not a consequence of the detailed assumptions about the TF-DNA interaction, as we tried to show by presenting first the "back of the envelope" arguments of Sec. II. While our approach was based on the fluctuation-dissipation theorem, we can also imagine extending the first passage time methods to arrive at the noise associated with diffusion: in calculating the "association rates," for which the methods of the mean first passage time are well developed, one is really computing the mean association rates. For Poisson processes, the variance in the flux due to diffusion scales with the mean arrival rate, but in general this is not true, and the behavior of the noise is a separate question. Extending the mean first passage time formalism to give results on the variance would thus allow for an independent calculation of noise.

The diffusive contribution to the total noise in gene expression has only recently been recognized as significant [13]. Metzler discusses the concept of "interaction volume" around the regulatory site on the DNA and studies the probability that transcription factors will enter this volume in the case of the $\lambda$-phage infecting Escherichia coli bacterium, concluding that the spatial fluctuations can be important in genetic circuits [28]. Holcman et al. study the masterdiffusion equation in the context of signaling molecules binding to and unbinding from ion channels [29]. The analysis of Bicoid-Hunchback system in the development of the fruit fly Drosophila melanogaster shows that the measured signatures in the noise in Hunchback expression are consistent with diffusive fluctuations in Hunchback's regulator, Bicoid [30,31]. van Zon and co-workers [32] study the diffusional component of the noise in Green's function reaction dynamics (GFRD) stochastic simulations and conclude by noting that $1 \mathrm{D}$ sliding along the DNA could have important effects on the noise power spectra. All these results point to the basic physics of diffusion as setting a limit to precision of a fundamental biological process, transcriptional regulation. Our results indicate that this fundamental limit is not easily evaded by the (still largely unknown) complexities of protein motion along the DNA molecule.

\section{ACKNOWLEDGMENTS}

We thank T. Gregor for helpful comments on the paper. This work was supported in part by NIH Grants No. P50 GM071508 and No. R01 GM077599, by NSF Grant No. PHY-0650617, and by the Burroughs Wellcome Fund Program in Biological Dynamics (G.T.). We thank the Center for Studies in Physics and Biology at Rockefeller University for its hospitality. 
[1] This limit is analogous to that identified long ago in the context of bacterial chemotaxis: H. C. Berg and E. M. Purcell, Biophys J. 20, 193 (1977). For a generalization of the BergPurcell argument, see Refs. [13,14]. For evidence that real transcriptional control systems approach this limit, see Refs. $[30,31]$.

[2] A. D. Riggs, S. Bourgeois, and M. Cohn, J. Mol. Biol. 53, 401 (1970).

[3] O. G. Berg, R. B. Winter, and P. H. von Hippel, Biochemistry 20, 6929 (1981).

[4] O. G. Berg and C. Blomberg, Biophys. Chem. 4, 367 (1976).

[5] O. G. Berg and C. Blomberg, Biophys. Chem. 7, 33 (1977).

[6] M. Slutsky and L. A. Mirny, Biophys. J. 87, 4021 (2004).

[7] D. M. Gowers, G. G. Wilson, and S. E. Halford, Proc. Natl. Acad. Sci. U.S.A. 102, 15883 (2005).

[8] H. Kabata, O. Kurosawa, I. Arai, M. Washizu, S. A. Margarson, R. E. Glass, and N. Shimamoto, Science 262, 1561 (1993).

[9] Y. M. Wang, R. H. Austin, and E. C. Cox, Phys. Rev. Lett. 97, 048302 (2006).

[10] J. Elf, G. W. Li, and X. S. Xie, Science 316, 1191 (2007).

[11] Several colleagues have presented this intuitive argument as a response to the discussion in Ref. [30], which claims that the observed precision of transcriptional regulation in the early fruit fly embryo is close the physical limit. We take this opportunity to thank those who insisted on a more rigorous answer.

[12] E. M. Lifshitz and L. P. Pitaevskii, Statistical Physics Part 2 (Pergamon, Oxford, 1980), Sec. 89.

[13] W. Bialek and S. Setayeshgar, Proc. Natl. Acad. Sci. U.S.A. 102, 10040 (2005).

[14] W. Bialek and S. Setayeshgar, Phys. Rev. Lett. 100, 258101 (2008).

[15] O. G. Berg and P. H. von Hippel, Annu. Rev. Biophys. Biophys. Chem. 14, 131 (1985).

[16] In case of transcriptional control, where the regulated protein is the "readout" of the transcription factor concentration, the integration time is usually the regulated protein lifetime (minutes or more); during this time the specific site occupancy will have equilibrated, implying that many translocation-related steps (binding and unbinding transitions to or from the specific site and the sliding state) occur during one integration time.

[17] Diffusion-limited reaction rates generally take the form $k_{+}$ $=4 \pi D L$, where $L$ is the effective target size that is primarily determined by the largest linear extent of the target. See Ref. [15] for details.

[18] N. Shimamoto, J. Biol. Chem. 274, 15293 (1999).

[19] I. M. Sokolov, R. Metzler, K. Pant, and M. C. Williams, Biophys. J. 89, 895 (2005).

[20] M. Coppey, O. Bénichou, R. Voituriez, and M. Moreau, Biophys. J. 87, 1640 (2004).

[21] M. A. Lomholt, T. Ambjörnsson, and R. Metzler, Phys. Rev. Lett. 95, 260603 (2005).

[22] S. E. Halford and J. F. Marko, Nucleic Acids Res. 32, 3040 (2004).

[23] O. G. Berg, Biopolymers 18, 2861 (1979).

[24] M. B. Elowitz, M. G. Surette, P. E. Wolf, J. B. Stock, and S. Leibler, J. Bacteriol. 181, 197 (1999).

[25] T. Gregor, E. F. Wieschaus, A. P. McGregor, W. Bialek, and D. W. Tank, Cell 130, 141 (2007).

[26] P. Singer and C. W. Wu, J. Biol. Chem. 262, 14178 (1987).

[27] P. C. Blainey, A. M. van Oijen, A. Banerjee, G. L. Verdine, and X. Sunney Xie, Proc. Natl. Acad. Sci. U.S.A. 103, 5752 (2006).

[28] R. Metzler, Phys. Rev. Lett. 87, 068103 (2001).

[29] D. Holcman and Z. Schuss, J. Chem. Phys. 122, 114710 (2005)

[30] T. Gregor, D. W. Tank, E. F. Wieschaus, and W. Bialek, Cell 130, 153 (2007).

[31] G. Tkačik, T. Gregor, and W. Bialek, PLoS ONE 3, e2774 (2008).

[32] J. S. van Zon, M. J. Morelli, S. Tanase-Nicola, and P. R. ten Wolde, Biophys. J. 91, 4350 (2006). 